\title{
Second Trimester Fetal and Maternal Epicardial Fat Thickness in Gestational Diabetic Pregnancies
}

Authors

Affiliations

\section{A. Yavuz ${ }^{1}$, M. O. Akkurt ${ }^{1}$, S. Yalcin ${ }^{1}$, G. Karakoc ${ }^{2}$, E. Varol ${ }^{3}$, M. Sezik}

${ }^{1}$ Division of Perinatology, Department of Obstetrics and Gynecology, Suleyman Demirel University, Faculty of Medicine Isparta, Turkey

${ }^{2}$ Division of Perinatology, Department of Obstetrics and Gynecology, Sifa University School of Medicine, Izmir, Turkey

${ }^{3}$ Department of Cardiology, Suleyman Demirel University, Faculty of Medicine, Isparta, Turkey
Key words

- epicardial fat thickness

- fetal echocardiography

gestational diabetes mellitus

- insulin resistance received 15.02 .2016

accepted 28.06.2016

\section{Bibliography}

DOI http://dx.doi.org/

10.1055/s-0042-111435

Published online:

July 29, 2016

Horm Metab Res 2016;

48: 595-600

(c) Georg Thieme Verlag KG

Stuttgart · New York

ISSN 0018-5043

\section{Correspondence}

\section{Sezik}

Division of Perinatology Department of Obstetrics and Gynecology

Suleyman Demirel University

School of Medicine

32260 Cunur

Isparta

Turkey

Tel.: + 90/246/2119183

Fax: + 90/246/2120383

msezik@yahoo.com

\section{Abstract \\ $\nabla$}

Our aim was to evaluate the association between gestational diabetes mellitus and sonographically measured fetal epicardial fat thickness between 24-28 weeks' gestation. This was a cross-sectional study that included 40 pregnancies with gestational diabetes mellitus, matched with 40 normal pregnancies with similar maternal age, body-mass index, gestational age, fetal gender, and fetal abdominal circumference on ultrasound. Fetal epicardial fat thickness was measured and recorded during ultrasonography at 24-28 weeks of gestation. Maternal evaluation included measurement of maternal epicardial fat thickness, using echocardiography. Fetal and maternal epicardial fat thickness values were compared across the groups. Ultrasound views of fetal epicardial fat thickness were evaluated independently by 3 perinatology fellows to determine inter- and intra-observer variability. Partial and intraclass correlation analyses were

\section{Introduction \\ $\nabla$}

Epicardial fat (EF) is a visceral fat tissue of the heart and derives from the brown fat tissue during embryogenesis, bearing a protective effect against cardiac hypothermia $[1,2]$. The protective mechanism is by absorbing free fatty acids when these are high in the circulation and by working as an energy source when energy need is increased [3]. EF tissue is located between the myocardium and the visceral pericardium, and is directly connected to the myocardium $[1,4]$. As there is no fascia separating EF tissue and myocardium, they share the same microcirculation [5]. EF is metabolically very active with secretion of many proinflammatory and proatherogenic cytokines including vasoactive peptides related with obesity, hypertension, and coronary heart used. Fetal and maternal epicardial fat thickness measurements were moderately correlated $(\mathrm{r}=0.63)$. Mean fetal and maternal epicardial fat thickness values were higher in gestational diabetes mellitus pregnancies $(\mathrm{p}=0.004$ and $\mathrm{p}<0.0001$, respectively) compared to controls. Fetal epicardial fat thickness was positively correlated $(r=0.43)$ with postchallenge 2 -h glucose values. Inter- and intra-observer agreement was high, demonstrated by strong correlations $(r=0.99$ and $r=0.99$, respectively) across fetal epicardial fat thickness measurements of the examiners. Fetuses from gestational diabetes mellitus pregnancies have significantly higher fetal and maternal epicardial fat thickness values compared to nongestational diabetes mellitus pregnancies. Fetal epicardial fat thickness obtained during second trimester fetal anatomy ultrasound may potentially be a reliable indicator for gestational diabetes mellitus. However, clinical validation studies are needed.

disease. The secretory activity includes interleukin 6, tumor necrosis factor alpha, angiotensin II, plasminogen activator, omentin, and nerve growth factor [6,7]. Moreover, EF tissue also secretes anti-inflammatory and antiaterogenic adipokins such as adiponectin and adrenomedullin [8].

To our knowledge, echocardiographic epicardial fat thickness (EFT) measurement in adults was first attempted by lacobellis et al. $[9,10]$ and was reported to vary between $1-23 \mathrm{~mm}$ [10]. Becaue EFT measurement seems to be unaffected by variations in skin and muscular tissue layers, it may show lipoidosis much more accurate than waist measurement [11]. Previous data from adult subjects indicate that EFT measured during echocardiography is in close relation with abdominal fat 
tissue measured via magnetic resonance imaging and computerized tomography [9].

EFT was reported to be increased in insulin resistance and in diabetes mellitus [12]. Gestational diabetes mellitus (GDM) is a metabolic disease manifesting itself as insulin resistance during pregnancy [6]. Diagnosis of GDM is important, as it causes an increase in maternal, fetal, and neonatal complications [13-15]. Insulin resistance develops long before fasting blood glucose levels increase, and the high insulin levels in blood is associated with lipogenesis and atherosclerosis $[16,17]$.

Although an association between sonographic EFT measurements and insulin resistance in adults has been previously shown, data on fetal EFT and GDM are scarce. Moreover, the relationship across fetal and maternal EFT measurements and the reliability of this parameter during midtrimester ultrasound has not been adequately investigated. We hypothesized that EFT measured during fetal ultrasound scans at 24-28 weeks of gestation has an acceptable reproducibility and a potential to predict maternal insulin resistance. For this purpose we compared and correlated fetal EFT values, maternal EFT values, and blood glucose measurements obtained during a $75 \mathrm{~g}$ oral glucose tolerance test (OGTT). We also evaluated inter- and intra-observer variability for fetal EFT measurements from recorded images.

\section{Subjects and Methods}

$\nabla$

The study protocol was subject to local ethics committee approval. Pregnant women scheduled for a $75 \mathrm{~g}$ OGTT at 24+0/6 to $28+0 / 6$ weeks of gestation between May 2015-December 2015 were included. The study group consisted of 40 women with GDM diagnoses according to the American Diabetes Association criteria of $75 \mathrm{~g}$ OGTT results. The diagnosis of GDM was made when any one of the following plasma glucose values was exceeded: fasting $\geq 92 \mathrm{mg} / \mathrm{dl}, 1 \mathrm{~h} \geq 180 \mathrm{mg} / \mathrm{dl}$, or $2 \mathrm{~h} \geq 153 \mathrm{mg} / \mathrm{dl}$ [18]. Those with pre-existing type 1 or type 2 diabetes were excluded. In the control group, there were 40 pregnancies with normal $75 \mathrm{~g}$ OGTT values, matched for maternal age, body-mass index (BMI), gestational age, fetal gender, and fetal abdominal circumference $(A C)$ on ultrasound. Demographic data were extracted from patient files. Gestational age depended on the last menstrual period confirmed by a first trimester crownlength measurement. All women were weighed and their height measured at the time of OGTT, and BMI was calculated. Fetal anatomy scan including cardiac examination and maternal echocardiography were also performed on the same day as the OGTT. Those with fetal anomalies, accompanying maternal disease (except GDM), and women on medications other than oral iron supplementation and/or multivitamins had been excluded. Fetal and maternal EFT evaluations were performed by 3 perinatology fellows with a Voluson E6 ultrasound equipment (General Electric, Tiefenbach, Austria) fitted with a 2-7 MHz convex abdominal probe and with a TTE VIVID 7 (General Electric, USA) ultrasound device in the adult cardiology unit of our hospital by a cardiologist experienced in adult echocardiography, respectively. As previously described by Iacobellis [9], measurements were made at end-diastole in 3 cardiac cycles through the available wall of the right ventricle both in the fetus and the mother ( $\odot$ Fig. 1). As a benchmark, the highest EFT measured throughout 3 cycles from the perpendicular wall of the right ventricle across the ultrasound ray vertical to the aortic annulus was recorded [9]. All the images that included fetal EFT measurements were saved as JPEG files.

Following retrieval of all images, the saved ultrasound views were evaluated independently by the same 3 perinatology fellows to determine interobserver variability. The evaluations were repeated following a period of 2 weeks to establish intraobserver variability. Intraclass correlation coefficients were calculated to determine the inter- and intra-observer agreement during first and second occasions.

\section{Statistical analysis}

Data are expressed as mean \pm standard deviations (SD). Kolmogorov-Smirnov test was used to determine the distribution of variables. Mann-Whitney U-test and chi-square test were used for comparisons. Partial correlation analyses controlling for confounding variables such as maternal age, gestational weeks, BMI and $\mathrm{AC}$ measurement on ultrasound were used to determine the relationship between clinical parameters and EFT. A p-value $<0.05$ was considered significant in all analyses. Corre-
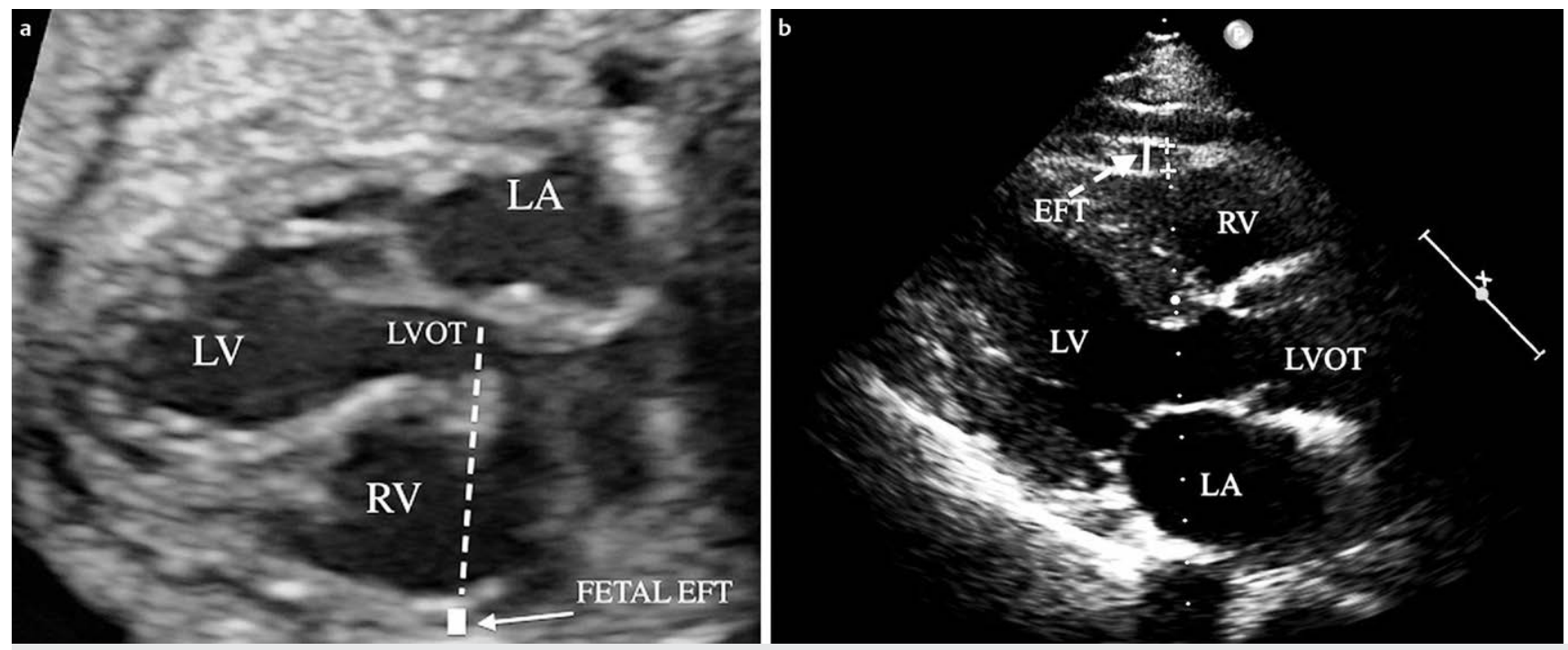

Fig. 1 The distance on the line running to the aortic annulus between epicardium and myocardium is described as an epicardial thickness, shown in the fetal a and maternal b echocardiographic views (LA: left atrium; LV: left ventricle; LVOT: left ventricle outflow tract; RV: right ventricle). 


\begin{tabular}{|lccll} 
& Control Group & GDM Group & p-Value & $\begin{array}{l}\text { Table } 1 \text { Comparisons of baseline } \\
\text { and output data in gestational } \\
\text { diabetic and control groups. }\end{array}$ \\
\hline Maternal age (years) & $27.8 \pm 6.3$ & $27.6 \pm 6.3$ & 0.888 & 0.171 \\
\hline Body-mass index $\left(\mathrm{kg} / \mathrm{m}^{2}\right)$ & $27.5 \pm 2.7$ & $28.3 \pm 2.8$ & 0.880 \\
\hline Gestational age (weeks) & $25.9 \pm 1.2$ & $26.0 \pm 1.2$ & 0.117 \\
\hline Fetal gender (male/female) & $1.5(24 / 16)$ & $0.74(17 / 23)$ & 0.413 \\
\hline Estimated AC on ultrasound $(\mathrm{mm})$ & $219.9 \pm 14.1$ & $222.5 \pm 15.0$ & 0.004 \\
\hline Fetal EFT (mm) & $1.31 \pm 0.03$ & $1.34 \pm 0.04$ & 0.0001 \\
\hline Maternal EFT (mm) & $5.3 \pm 1.3$ & $6.9 \pm 1.6$ & \\
\hline OGTT $(\mathrm{mg} / \mathrm{dl})$ & & & 0.0001 \\
\hline Fasting & $80.9 \pm 5.0$ & $90.3 \pm 8.0$ & 0.0001 \\
\hline Hour 1 & $163.1 \pm 5.3$ & $184.9 \pm 7.0$ & 0.0001 \\
\hline Hour 2 & $125.2 \pm 5.8$ & $148.9 \pm 7.1$ & \\
\hline
\end{tabular}

Data are expressed as mean \pm standard deviations or ratios. AC: Abdominal circumference; EFT: Endocardial fat thickness;

GDM: Gestational diabetes; OGTT: Oral glucose challenge test

lation coefficients ( $\mathrm{r}$ ) between $0.3-0.7$ indicated moderate and $>0.7$ strong correlations, respectively.

\section{Results}

The GDM and control groups $(n=40$, each) were homogenous concerning maternal age, maternal BMI, gestational weeks, fetal ultrasound AC estimation, and fetal gender ( $p>0.05$ for all comparisons). As expected, fasting, 1-h, and 2-h OGTT serum glucose values were significantly higher $(p<0.0001$ for all comparisons) in women with GDM compared to the controls. In the GDM group, fetal $(\mathrm{p}=0.004)$ and maternal EFT $(\mathrm{p}<0.0001)$ were found to be significantly increased ( $\odot$ Table 1, $\odot$ Fig. 2 ).

- Table 2 summarizes the result of correlations after controlling for confounding factors such as maternal age, gestational weeks at ultrasound, maternal obesity, and fetal macrosomia. Maternal and fetal EFT measurements were moderately and positively associated. In the whole group, there were significant correlations across maternal EFT and all the $75 \mathrm{~g}$ OGTT results ( $\bullet$ Table 2). These associations were present, but weaker at 1 -h and 2-h results considering fetal EFT. When only GDM pregnancies were included, maternal EFT was no longer independently correlated with OGTT results, whereas fetal EFT was positively and moderately related to 2 -h glucose values ( $\bullet$ Table 2 ). These data indicate that fetal EFT is associated with increased 2-h postchallenge serum glucose measurements, independent of maternal obesity and fetal size in pregnancies with GDM.

- Table 3 and $\odot$ Fig. 3 show the results of intraclass correlation analyses for the inter- and intra-observer agreement of fetal EFT from prerecorded ultrasound images across 3 perinatology research fellows. The correlation coefficients were high $(r=0.99$, $\mathrm{p}<0.0001$ and $\mathrm{r}=0.99, \mathrm{p}<0.0001)$, indicating high inter- and intra-observer agreements ( Table 3, $\bullet$ Fig. 3). Bland-Altman plots with their respective $95 \%$ limits of agreement across observers are provided in $\bullet$ Fig. 4.

\section{Discussion and Conclusions \\ $\nabla$}

In our study, ultrasound estimation of EFT was significantly higher in gestational diabetic pregnancies compared to normal pregnancies. While the mean difference in EFT measurement was relatively small $(0.02 \mathrm{~mm})$, this was statistically significant. Although the clinical application of such difference requires further validation studies, to our knowledge ours is the first pro-

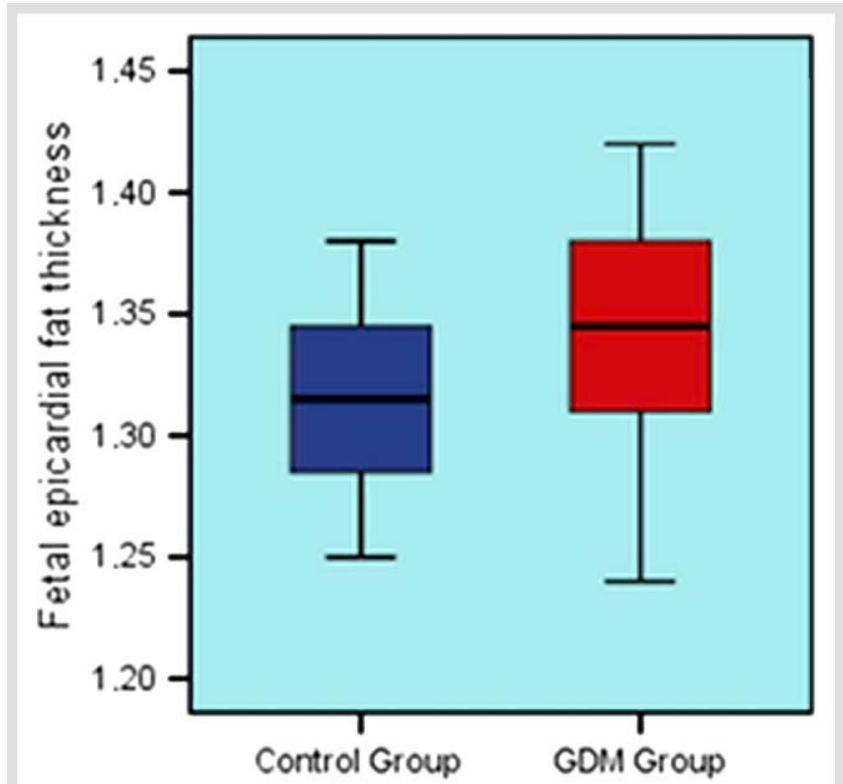

Fig. 2 Fetal epicardial fat thickness values in the study and control groups. (Color figure available online only).

spective study, in which fetal EFT was measured in women with GDM.

EFT measurement with transthoracic echocardiography in adults has recently been a popular study topic. Increased EFT has been found in relation with metabolic syndrome and coronary heart diseases in many studies $[9,12,19,20]$. Previous publications revealed a significant relationship between EFT, fasting blood glucose, and DM in adults [12,21,22].

Some other studies investigated the relationship across EFT and glucose intolerance in women during or after gestational diabetic pregnancies. In 62 women with previous GDM, EFT measured by echocardiography was significantly increased compared to the control group [23]. The authors stated that high EFT results might indicate presence of atherosclerosis in women with previous GDM [23]. In another study, mean EFT was measured as $7.2 \mathrm{~mm}$ in pregnant women with GDM vs. $5.6 \mathrm{~mm}$ in controls, revealing a significant increment. In the same study, significant correlations between EFT, BMI, and postprandial serum glucose levels were also present [11]. These findings were supported by another investigation indicating that postprandial glucose and BMI were associated with maternal EFT in regression models [24]. Our data showing higher maternal EFT meas- 


\begin{tabular}{|c|c|c|c|c|c|c|c|}
\hline & & & \multirow[t]{2}{*}{ Fetal EFT } & \multirow[t]{2}{*}{ Maternal EFT } & \multicolumn{3}{|c|}{$75 \mathrm{~g}$ OGTT results } \\
\hline & & & & & Fasting & Hour 1 & Hour 2 \\
\hline \multirow{4}{*}{$\begin{array}{l}\text { Overall } \\
(n=80)\end{array}$} & \multirow{2}{*}{$\begin{array}{c}\text { Fetal EFT } \\
(\mathrm{mm})\end{array}$} & $r$ & - & 0.627 & 0.238 & 0.347 & 0.401 \\
\hline & & $\mathrm{p}$ & - & 0.0001 & 0.238 & 0.038 & 0.0001 \\
\hline & \multirow{2}{*}{$\begin{array}{l}\text { Maternal EFT } \\
\qquad(\mathrm{mm})\end{array}$} & $r$ & 0.627 & - & 0.425 & 0.557 & 0.576 \\
\hline & & $\mathrm{p}$ & 0.0001 & - & 0.0001 & 0.0001 & 0.0001 \\
\hline \multirow{4}{*}{$\begin{array}{l}\text { GDM group } \\
(n=40)\end{array}$} & \multirow{2}{*}{$\begin{array}{c}\text { Fetal EFT } \\
(\mathrm{mm})\end{array}$} & $r$ & - & 0.463 & -0.204 & -0.019 & 0.428 \\
\hline & & $\mathrm{p}$ & - & 0.004 & 0.232 & 0.913 & 0.009 \\
\hline & \multirow{2}{*}{$\begin{array}{l}\text { Maternal EFT } \\
(\mathrm{mm})\end{array}$} & $r$ & 0.463 & - & -0.025 & 0.029 & 0.227 \\
\hline & & $p$ & 0.004 & - & 0.884 & 0.868 & 0.182 \\
\hline
\end{tabular}

Table 2 Correlations across fetal and maternal epicardial fat thickness and $75 \mathrm{~g}$ oral glucose tolerance test results, after controlling for maternal age, gestational age, body-mass index, and abdominal circumference on ultrasound.

EFT: Endocardial fat thickness; GDM: Gestational diabetes; OGTT: Oral glucose challenge test; r: Adjusted correlation coefficient by partial correlation analysis

\begin{tabular}{|c|c|c|c|c|c|}
\hline & \multicolumn{5}{|c|}{ Fetal epicardial fat thickness (mm) } \\
\hline & Range & Median & Mean \pm SD & $\mathbf{r}$ & $\mathbf{p}$ \\
\hline \multicolumn{6}{|l|}{ First occasion } \\
\hline Observer I & $1.24-1.38$ & 1.32 & $1.32 \pm 0.03$ & 0.990 & 0.0001 \\
\hline Observer II & $1.24-1.38$ & 1.33 & $1.32 \pm 0.03$ & & \\
\hline Observer III & $1.24-1.38$ & 1.32 & $1.32 \pm 0.03$ & & \\
\hline \multicolumn{6}{|c|}{ Second occasion } \\
\hline Observer I & $1.23-1.37$ & 1.32 & $1.32 \pm 0.03$ & 0.986 & 0.0001 \\
\hline Observer II & $1.23-1.37$ & 1.33 & $1.32 \pm 0.03$ & & \\
\hline Observer III & $1.24-2.38$ & 1.32 & $1.32 \pm 0.03$ & & \\
\hline
\end{tabular}

Table 3 Intraclass correlation analyses for inter- and intra-variability between the first and second fetal epicardial fat thickness measurements by 3 research fellows.
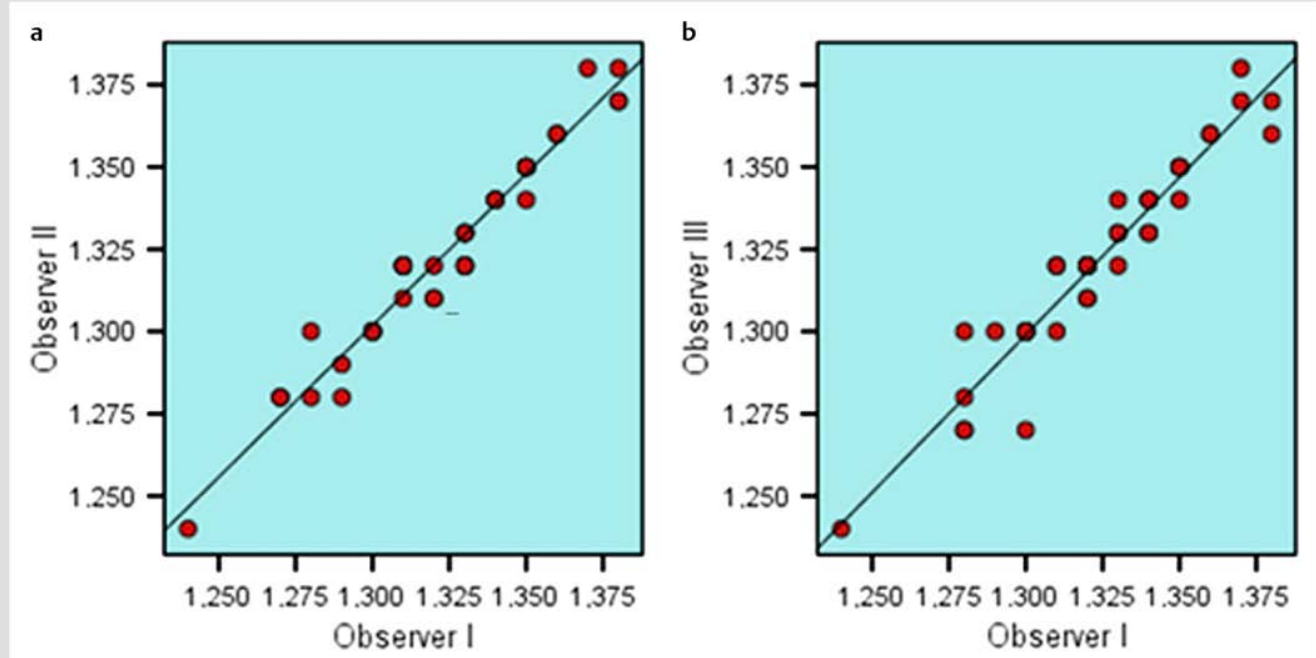

Fig. 3 Plots showing correlation among 3 perinatology research fellows for the measurement of fetal epicardial fat thickness. (Color figure available online only).

urements in pregnancies with GDM (6.9 vs. $5.3 \mathrm{~mm}$ ) support these findings. Although we showed significant correlations across maternal EFT and OGTT values in the overall study group, these associations disappeared when only the gestational diabetics were taken into account. This may be due to limited number of GDM women in our design or lack of an association, as we rigorously controlled for confounding factors such as maternal age, maternal obesity, and fetal size. Therefore, fetal but not maternal EFT may be an independent predictor of postchallenge serum glucose levels.

The effect of gender on EFT has not been adequately studied. Some investigators reported a gender difference in adults, with increased measurements in men $(7.6 \mathrm{~mm}$ vs. $6.9 \mathrm{~mm}$ ) [9]. In our study, fetal EFT values were similar in male and female fetuses. These preliminary data may indicate an environmental effect on
EFT considering gender, rather than nonimprinted genetic influences.

Recently, Jackson et al. retrospectively performed fetal EFT measurements in the second trimester in 28 diabetic and 28 nondiabetic women. After controlling for maternal BMI, estimated fetal weight, birth weight, fetal abdominal circumference, and subcutaneous fat thickness, EFT was significantly higher in diabetic $(1.43 \mathrm{~mm})$ vs. control fetuses $(1.16 \mathrm{~mm})$. To our knowledge, this was the first study on fetal EFT measurements in diabetic pregnancies [25]. However, a limitation was its retrospective design not allowing estimating the EFT value by measuring through 3 cardiac cycles during end-diastole as originally described by Iacobellis. We aimed to overcome such limitation by prospectively performing both maternal and fetal EFT measurements, using a standardized technique. In this context, ours 

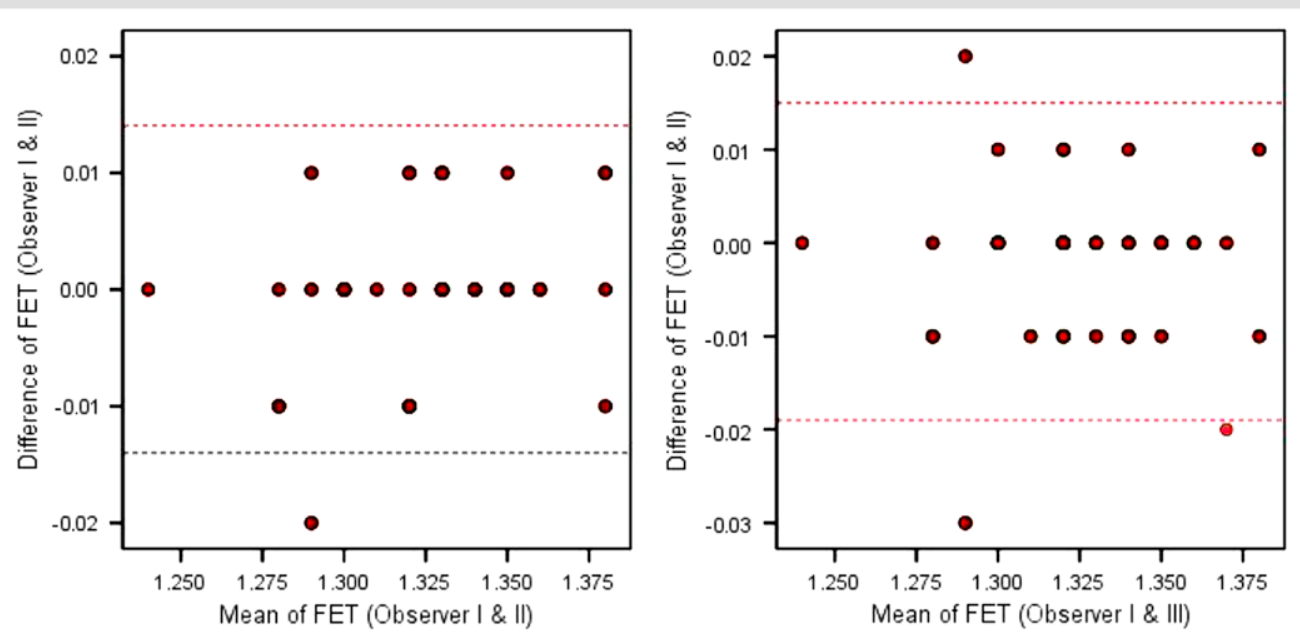

Fig. 4 Bland-Altman plots with their respective $95 \%$ limits of agreement across observers. (Color figure available online only).

is probably the first prospective study that includes measurements of EFT from gestational diabetic pregnancies and corroborates previous findings by Jackson et al. despite a lower difference in measured values.

Depending on our results, fetal EFT measurement seems to be a novel noninvasive parameter for the metabolic status of the pregnancy. On the other hand, EFT estimation requires high definition ultrasonography and medical staff specialized on fetal echocardiography. Fetal EFT measurement is also difficult to perform when the heart is in posterior position, especially during early gestational weeks. Therefore, it should not currently be considered as a routine screening test. However, fetal EFT measurements can be performed during detailed fetal ultrasonograms of high-risk pregnancies at specialized centers. This may allow determination of pregnancies requiring maternal dietary and physical activity interventions. Moreover, studies evaluating neonatal and long-term outcomes of fetuses with increased EFT values should be encouraged.

Some drawbacks of our study include relatively limited number of patients and its cross-sectional design that does not allow follow-up through delivery or neonatal period. We did not include maternal serum insulin and glycated hemoglobin measurements. Another drawback was lack of storage as video clips, which is a preferable method to static images for the assessment of intra- and inter-observer reproducibility. This may have led to overestimation of intra- and inter-observer correlation results in our design. Nevertheless, we have provided robust prospective data on this subject, including reliability of the fetal EFT measurements. Further large-scaled prospective follow-up studies with clinical validation trials on the feasibility of fetal and maternal EFT evaluations in pregnancies with metabolic syndrome are necessary.

\section{Statement}

$\nabla$

The investigation was carried out at Suleyman Demirel University, Faculty of Medicine, Department of Obstetrics and Gynecology, Division of Perinatology, Isparta, Turkey.

Abstract accepted as an oral presentation at the $26^{\text {th }}$ World Congress on Ultrasound in Obstetrics and Gynecology, 25-28 September 2016, Rome, Italy.

\section{Conflict of Interest}

$\nabla$

The authors declare no conflict of interest.

\section{References}

1 Kremen J, Dolinkova M, Krajickova J, Blaha J, Anderlova K, Lacinova Z, Haluzikova D, Bosanska L, Vokurka M, Svacina S, Haluzik M. Increased subcutaneous and epicardial adipose tissue production of proinflammatory cytokines in cardiac surgery patients: possible role in postoperative insulin resistance. J Clin Endocrinol Metab 2006; 91: 4620-4627

2 Sacks HS, Fain JN, Holman B, Cheema P, Chary A, Parks F, Karas J, Optican R, Bahouth SW, Garrett E, Wolf RY, Carter RA, Robbins T, Wolford $D$, Samaha J. Uncoupling protein-1 and related messenger ribonucleic acids in human epicardial and other adipose tissues: epicardial fat functioning as brown fat. J Clin Endocrinol Metab 2009; 94: 36113615

3 Marchington JM, Pond CM. Site specific properties of pericardial and epicardial adipose tissue: the effects of insulin and high-fat feeding on lipogenesis and the incorporation of fatty acids in vivo. Int J Obesity 1990; 14: 1013-1022

4 Cheng KH, Chu CS, Lee KT, Lin TH, Hsieh CC, Chiu CC, Voon WC, Lai WT. Adipocytokines and proinflammatory mediators from abdominal and epicardial adipose tissue in patients with coronary artery disease. Int J Obesity 2008; 32: 268-274

5 Iacobellis G, Willens HJ. Echocardiographic epicardial fat: A review of research and clinical applications. J Am Soc Echocardiogr 2009; 22: 1311-1319

6 Cetin M, Cakici M, Polat M, Suner A, Zencir C, Ardic I. Relation of epicardial fat thickness with carotid intima-media thickness in patients with type 2 diabetes mellitus. Int J Endocrinol 2013; 2013: 769175

7 Altun B, Tasolar H, Eren N. Epicardial adipose tissue thickness in hemodialysis patients. Echocardiography 2014; 31: 941-946

8 Teijeira-Fernandez E, Eiras S, Grigorian-Shamagian L, Fernandez A, Adrio B, Gonzalez-Juanatey JR. Epicardial adipose tissue expression of adiponectin is lower in patients with hypertension. J Hum Hypertens 2008; 22: 856-863

9 Iacobellis G, Ribaudo MC, Assael F, Vecci E, Tiberti C, Zappaterreno A, Di Mario U, Leonetti F. Echocardiographic epicardial adipose tissue is related to anthropometric and clinical parameters of metabolic syndrome: a new indicator of cardiovascular risk. J Clin Endocrinol Metab 2003; 88: 5163-5168

10 Iacobellis G, Assael F, Ribaudo MC, Zappaterreno A, Alessi G, Di Mario $U$, Leonetti $F$. Epicardial fat from echocardiography: a new method for visceral adipose tissue prediction. Obes Res 2003; 11: 304-310

11 Jeong JW, Jeong MH, Yun KH, Oh SK, Park EM, Kim YK. Echocardiographic epicardial fat thickness and coronary artery disease. Circ J 2007; 71: 536-539

12 Ahn SG, Lim HS, Joe DY, Kang SJ, Choi BJ, Choi SY, Yoon MH, Hwang GS, Tahk SJ, Shin JH. Relationship of epicardial adipose tissue by echocardiography to coronary artery disease. Heart 2008; 94: e7 
13 American Diabetes Association. Diagnosis and classification of diabetes mellitus. Diabetes Care 2009; 32: 62-67

14 Noussitou P, Monbaron D, Vial Y, Gaillard RC, Ruiz J. Gestational diabetes mellitus and the risk of metabolic syndrome: a population based study in Lausanne, Switzerland. Diabetes Metab 2005; 31: 361-369

15 König AB, Junginger S, Reusch J, Louwen F, Badenhoop K. Gestational Diabetes Outcome in a Single Center Study: Higher BMI in Children After Six Months. Horm and Metab Res 2014; 46: 804-809

16 Asemi Z, Karamali M, Esmaillzadeh A. Favorable Effects of Vitamin D Supplementation on Pregnancy Outcomes in Gestational Diabetes: A Double Blind Randomized Controlled Clinical Trial. Horm and Metab Res 2015; 47: 565-570

17 Drynda R, Peters CJ, Jones PM, Bowe JE. The Role of Non-Placental Signals in the Adaptation of Islets to Pregnancy. Horm and Metab Res 2015; 47: 64-71

18 American Diabetes Association. Standards of medical care in diabetes-2013. Diabetes Care 2013; 36: S11-S66

19 Nakazato R, Dey D, Cheng VY, Gransar H, Slomka PJ, Hayes SW, Thomson LE, Friedman JD, Min JK, Berman DS. Epicardial fat volume and concurrent presence of both myocardial ischemia and obstructive coronary artery disease. Atherosclerosis 2012; 221: 422-426
20 Iacobellis G, Zaki MC, Garcia D, Willens HJ. Epicardial Fat in Atrial Fibrillation and Heart Failure. Horm and Metab Res 2014; 46: 587-590

21 Sengul C, Cevik C, Ozveren O, Oduncu V, Sunbul A, Akgun T, Can MM, Semiz E, Dindar I. Echocardiographic epicardial fat thickness is associated with carotid intima-media thickness in patients with metabolic syndrome. Echocardiography 2011; 28: 853-853

22 Iacobellis G, Barbaro G, Gerstein HC. Relationship of epicardial fat thickness and fasting glucose. Int J Cardiol 2008; 128: 424-426

23 Caliskan M, Caklili OT, Caliskan Z, Duran C, Ciftci FC, Avci E, Gullu H, Kulaksizoglu M, Koca H, Muderrisoglu $H$. Does gestational diabetes history increase epicardial fat and carotid intima media thickness? Echocardiography 2014; 31: 1182-1187

24 Nar G, Inci S, Aksan G, Unal OK, Nar R, Soylu K. The relationship between epicardial fat thickness and gestational diabetes mellitus. Diabetol Metab Syndr 2014; 6: 120

25 Jackson D, Deschamps D, Myers D, Fields D, Knudtson E, Gunatilake $R$. Fetal epicardial fat thickness in diabetic and non-diabetic pregnancies: A retrospective cross-sectional study. Obesity 2016; 24: 167-171 